\title{
Avaliação da atividade larvicida de saponinas triterpênicas isoladas de Pentaclethra macroloba (Willd.) Kuntze (Fabaceae) e Cordia piauhiensis Fresen (Boraginaceae) sobre Aedes aegypti
}

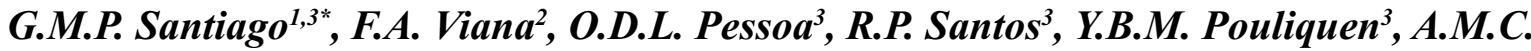 \\ Arriaga $^{3}$, M. Andrade-Neto ${ }^{3}$, R. Braz-Filho ${ }^{4}$
}

\begin{abstract}
${ }^{1}$ Departamento de Farmácia, Faculdade de Farmácia, Odontologia e Enfermagem, Universidade Federal do Ceará, Rua Capitão Francisco Pedro, 1210, Rodolfo Teófilo, 60430-370, Fortaleza, CE, Brasil, ${ }^{2}$ Departamento de Química, Universidade do Estado do Rio Grande do Norte, 59610-210, Mossoró, RN, Brasil, ${ }^{3}$ Departamento de Química Orgânica e Inorgânica, Universidade Federal do Ceará, CP 12200, 60021-970, Fortaleza, CE, Brasil, ${ }^{4}$ Setor de Química de Produtos Naturais, LCQUI, CCT, Universidade Estadual do Norte Fluminense, 28013-600, Campos, RJ, Brasil
\end{abstract}

\begin{abstract}
RESUMO: A atividade larvicida de quatro saponinas monodesmosídicas (1-4) isoladas de Pentaclethra macroloba e de uma saponina bidesmosídica (5) isolada de Cordia piauhiensis foi avaliada sobre larvas de estágio 3 de Aedes aegypti. As larvas foram expostas a várias concentrações (500, 250, 100, 50, 25 e 12,5 $\mu \mathrm{g} / \mathrm{mL}$ ) das saponinas por um período de $24 \mathrm{~h}$. Os resultados indicam que somente as saponinas 1-3 mostraram alta atividade larvicida, com $\mathrm{CL}_{50}$ variando de 18,6 a $27,9 \mu \mathrm{g} / \mathrm{mL}$. Estes resultados ressaltam as potencialidades destas saponinas como possíveis agentes larvicidas naturais.
\end{abstract}

Unitermos: Aedes aegypti, Pentaclethra macroloba, Cordia piauhiensis, saponinas.

\begin{abstract}
Evaluation of larvicidal activity of triterpenoid saponins isolated of Pentaclethra macroloba (Willd.) Kuntze (Fabaceae) and Cordia piauhiensis Fresen (Boraginaceae) against Aedes aegypti". The larvicidal activity of the four monodesmoside saponins (1-4) isolated from Pentaclethra macroloba and one bidesmoside saponin (5) from Cordia piauhiensis was evaluated on $3^{\text {rd }}$ instar larvae of Aedes aegypti. The larvae were exposed to serial concentrations (500, 250, 100, 50, 25 and $12.5 \mu \mathrm{g} / \mathrm{mL}$ ) saponins for a period of $24 \mathrm{~h}$. The results indicate that, only the saponins 1-3 showed high larvicidal activity, with $\mathrm{LC}_{50}$ ranging of 18,6-27,9 $\mu \mathrm{g} / \mathrm{mL}$. These results suggest that these can be used as natural larvicidal agents.
\end{abstract}

Keywords: Aedes aegypti, Pentaclethra macroloba, Cordia piauhiensis, saponins.

\section{INTRODUÇÃO}

Mosquitos atuam como vetores de diversas doenças, causando sérios problemas à saúde do homem e em alguns casos levando ao desenvolvimento de epidemias de difícil controle. O uso contínuo e indiscriminado de inseticidas químicos no combate a mosquitos causadores em potencial de certas doenças infecciosas tem provocado danos à saúde do homem e ao meio ambiente, além de promover a seleção de resistência nestes aos inseticidas comerciais. Estes efeitos indesejáveis têm instigado pesquisadores a buscar medidas alternativas de erradicação de mosquitos. Desta forma, pesquisas estão sendo realizadas no sentido de descobrir inseticidas naturais, efetivos e seguros. Muitas plantas produzem metabólitos secundários que apresentam atividade inibitória de crescimento de insetos (Chariandy et al., 1999), enquanto outras, agem como repelente (Mohan; Fields, 2002). O uso destes constituintes de plantas como agentes de controle de insetos e como controle de larvas é uma interessante perspectiva.

O Aedes aegypti atua como vetor da febre amarela na América Central, na América do Sul e no Oeste da África. Este mosquito é também o vetor da dengue, que é endêmica no Sudoeste da Ásia, ilhas do Pacífico e Américas (Ciccia; Cousio; Mongelli, 2000). A dengue é uma doença infecciosa causada por um arbovírus e transmitida de uma pessoa doente a uma pessoa sadia através da picada da fêmea contaminada.

Para se evitar a doença fez-se necessário 


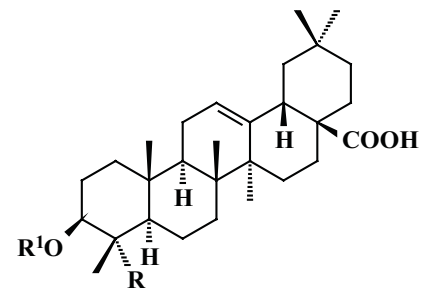

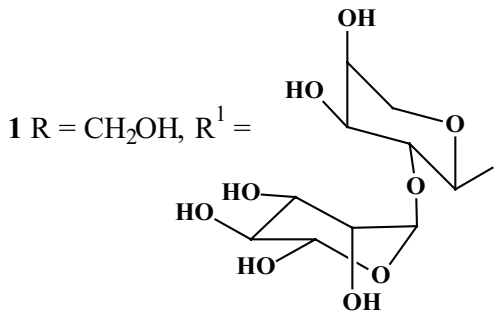

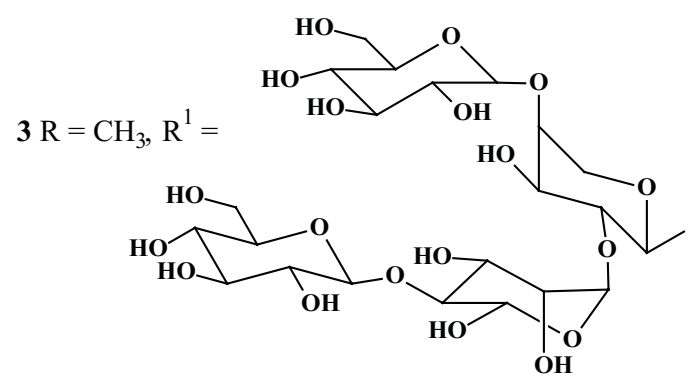

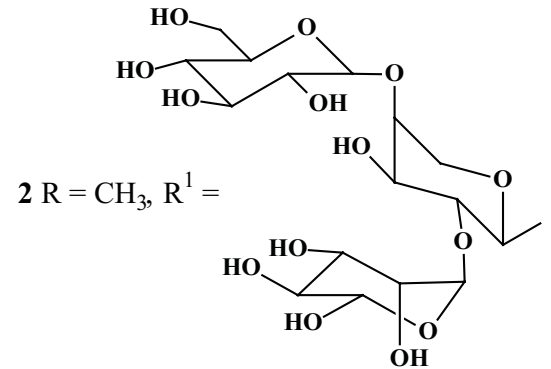

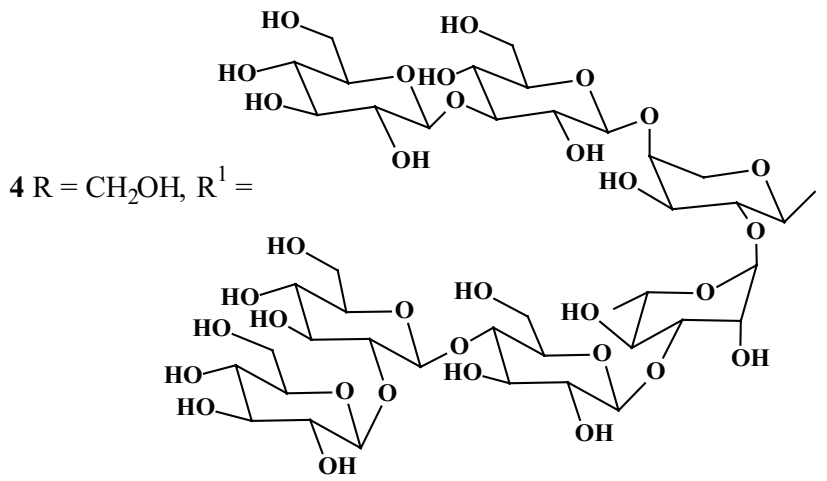

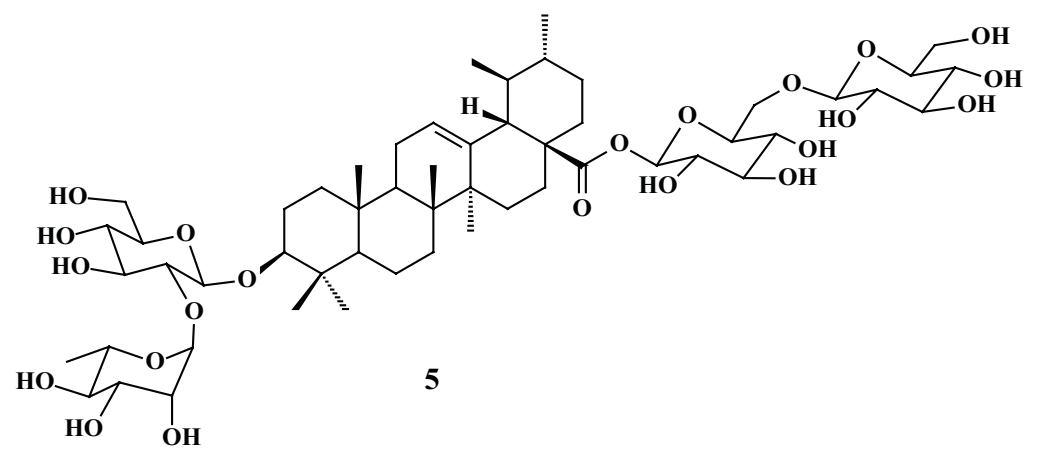

Figura 1. Estruturas das saponinas $\mathbf{1}$ - $\mathbf{5}$ testadas sobre as larvas de Aedes aegypti. As saponinas 1-4 foram isoladas de Pentaclethra macroloba e a saponina $\mathbf{5}$ foi isolada de Cordia piauhiensis.

intensificar as ações de prevenção e combate ao vetor da dengue.

Existem inúmeros trabalhos descrevendo o uso de produtos naturais (Yang et al., 2002; Araujo et al., 2003; Albuquerque et al., 2004), inclusive de saponinas como agentes larvicidas (Pelah; Abramovich; Wiesman, 2002).
Tendo em vista a ocorrência desta classe de substâncias químicas nas espécies Pentaclethra macroloba (Willd.) Kuntze (Fabaceae) e Cordia piauhiensis Fresen. (Boraginaceae), decidiu-se avaliar a atividade larvicida das saponinas isoladas a partir destas espécies vegetais.

$$
\text { A espécie Pentaclethra macroloba (Willd.) }
$$


Tabela 1. Valores de percentual da mortalidade das larvas de Aedes aegypti causadas pelas saponinas 1-5

\begin{tabular}{cccccc}
\hline Conc. $(\mu \mathrm{g} / \mathrm{mL})$ & $\mathbf{1}$ & $\mathbf{2}$ & $\mathbf{3}$ & $\mathbf{4}$ & $\mathbf{5}$ \\
\hline 500 & 100 & 100 & 100 & 100 & 0 \\
250 & 100 & 100 & 100 & 100 & 0 \\
100 & 100 & 100 & 100 & 40 & 0 \\
50 & 100 & 100 & 100 & 0 & 0 \\
25 & 89 & 51 & 16 & 0 & 0 \\
12,5 & 7 & 0 & 0 & 0 & 0 \\
\hline
\end{tabular}

Kuntze (Fabaceae), conhecida popularmente como “pracaxi” é encontrada na região Amazônica, especificamente no Estado do Amapá e estudos relatam a atividade inseticida de suas sementes (Chun et al., 1994), enquanto Cordia piauhiensis Fresen. (Boraginaceae) é uma planta endêmica do Brasil, distribuída nas regiões Sul, Sudeste, Sudoeste e Nordeste (Santos et al., 2003).

\section{MATERIAL E MÉTODOS}

\section{Material vegetal}

Galhos e sementes da espécie P. macroloba foram coletados na localidade de Fazendinha, no município de Macapá-AP, enquanto o caule de $C$. piauhiensis foi coletado no município do Crato-CE. As exsicatas das plantas representando estas coletas encontram-se arquivadas no Herbário Prisco Bezerra da Universidade Federal do Ceará, sob os números EAC-2594 e EAC 29104, respectivamente.

\section{Constituintes químicos}

As saponinas 1-4 foram isoladas e purificadas a partir dos extratos de P. macroloba: 1 foi isolada do extrato etanólico das sementes, $\mathbf{2}$ e $\mathbf{3}$ do extrato etanólico da casca dos galhos e a saponina 4 do extrato etanólico do lenho dos galhos. É válido acrescentar que o isolamento, a purificação e a determinação estrutural das saponinas 1-4 encontram-se descritos na literatura (Viana et al., 2004a,b). Por outro lado, a saponina 5 foi isolada do caule de $C$. piauhiensis (Santos et al., 2003).

No isolamento das saponinas $\mathbf{1 - 5}$ (Figura 1 ) foi empregado uma combinação de técnicas clássicas como cromatografia gravitacional em gel de sílica, cromatografia por exclusão molecular usando Sephadex LH-20, bem como, técnica de alta precisão e reprodutibilidade como cromatografia líquida de alta eficiência- CLAE.

$\mathrm{Na}$ elucidação estrutural utilizou-se técnicas espectroscópicas de RMN ${ }^{1} \mathrm{H}$ e ${ }^{13} \mathrm{C}$ uni- e bidimensionais $\left({ }^{1} \mathrm{H},{ }^{1} \mathrm{H}-\mathrm{COSY}\right.$, gs-HMQC, gs-HMBC, ROESY, NOESY e TOCSY)bem como, espectrometria demassa porionização química por eletrospray (ESI) e por bombardeamento rápido de átomos (FAB), fundamentais na análise da sequência de ligação dos açúcares nas unidades osídicas (Santos et al., 2003; Viana et al., 2004a,b).

\section{Ensaio larvicida}

Nos bioensaios, os ovos de A. aegypti foram eclodidos em água isenta de cloro, à temperatura ambiente. Em condições normais, os ovos maduros eclodem quando submersos em meio líquido e apresentam quatro estágios larvários, sendo selecionadas larvas de estágio 3 para serem utilizadas no presente estudo.

Em um béquer de $50 \mathrm{~mL}$, à temperatura ambiente, as amostras testadas, em diferentes concentrações (12,5 $\mu \mathrm{g} / \mathrm{mL}$ a $500 \mu \mathrm{g} / \mathrm{mL}$ ) foram dissolvidas em $0,3 \mathrm{~mL}$ de DMSO e a cada solução foram adicionadas 50 larvas de estágio 3, completando-se o volume para $20 \mathrm{~mL}$ com água. Após 24 horas, à temperatura ambiente, as larvas mortas foram contadas e calculada a percentagem letal e posteriormente a $\mathrm{CL}_{50}$. Os testes foram feitos em triplicata (Oliveira et al., 2002). Paralelamente foram feitos testes em branco, utilizando-se DMSO e água.

\section{RESULTADOS E DISCUSSÃO}

O potencial larvicida das saponinas 1-5 (Figura 1) foi avaliado sobre larvas do mosquito A. aegypti com o objetivo de encontrar produtos naturais eficazes e seguros e que no futuro possam substituir os compostos organofosforados ou outros agentes sintéticos.

A análise do percentual de mortalidade das larvas

Tabela 2. Valores de $\mathrm{CL}_{50}(\mu \mathrm{g} / \mathrm{mL})$ apresentados pelas saponinas 1, 2, 3 e 4 frentre às larvas de Aedes aegypti.

\begin{tabular}{cccc}
\hline Amostra testada & Número de açúcares & Genina & CL $_{50}(\mu \mathrm{g} / \mathrm{mL})$ \\
\hline $\mathbf{1}$ & 2 & hederagenina & $18,6 \pm 0,29$ \\
$\mathbf{3}$ & 3 & ácido oleanólico & $25,1 \pm 0,41$ \\
$\mathbf{4}$ & 4 & ácido oleanólico & $27,9 \pm 0,26$ \\
& 7 & hederagenina & $104,7 \pm 0,33$ \\
\hline & & & Rev. Bras. Farmacogn. \\
\hline & & Braz J. Pharmacogn. & 189 \\
& & 13 (3):jul/set. 2005
\end{tabular}


de A. aegypti, partindo de uma concentração de $500 \mu \mathrm{g} /$ $\mathrm{mL}$ a $12,5 \mu \mathrm{g} / \mathrm{mL}$ das saponinas $\mathbf{1 - 5}$ (Tabela 1), permitiu determinar os valores de $\mathrm{CL}_{50}$ (Tabela 2). Dentre as amostras que foram submetidas aos bioensaios, observouse que a saponina $\mathbf{1}$, a qual contém dois açúcares em sua unidade osídica apresentou a melhor atividade larvicida, com um valor de $\mathrm{CL}_{50}$ igual $18,6 \pm 0,29 \mu \mathrm{g} / \mathrm{mL}$. Foi também possível concluir que aquelas saponinas que continham um menor número de açúcares apresentaram uma melhor atividade (Tabela 1). Este fato tanto pode ser observado nas saponinas derivadas da hederagenina (1 e 4), como nas derivadas do ácido oleanóico ( 2 e 3 ). A saponina $\mathbf{5}$, que tem como genina o ácido ursólico não apresentou qualquer atividade larvicida.

É válido acrescentar que as saponinas 1-4, que apresentaram atividade larvicida, são monodesmosídicas e apresentam o grupo carboxila em C-28 livre, enquanto a saponina 5, que não apresentou atividade larvicida, é bidesmosídica e tem o seu grupo carboxila esterificado, sugerindo, portanto, que o grupo carboxila constitui uma unidade essencial para a atividade larvicida.

Tendo em vista os resultados obtidos, pode-se sugerir que a as saponinas $\mathbf{1}, \mathbf{2}$ e $\mathbf{3}$ são promissores agentes larvicidas, pois de acordo com a literatura (Cheng et al., 2003), substâncias com valores de $\mathrm{CL}_{50}$ menores que 100 $\mu \mathrm{g} / \mathrm{mL}$ são considerados bons agentes larvicidas.

\section{CONCLUSÃO}

O resultado da avaliação da atividade larvicida das saponinas 1-5 mostrou que a saponina 1, isolada das sementes de Pentaclethra macroloba, foi a mais ativa, confirmando, portanto, a atividade inseticida apresentada pelas sementes encontrada na literatura (Chun et al., 1994). Além do mais, observou-se um maior potencial larvicida para as saponinas monodesmosídicas e com um menor número de unidades de açúcares.

\section{AGRADECIMENTOS}

Os autores agradecem ao CNPq, CAPES, FUNCAP e PRONEX pelo suporte financeiro e ao NUEND pela concessão das larvas.

\section{REFERÊNCIAS}

Albuquerque MRJR, Silveira ER, Uchoa DEA, Lemos TLG, Souza ES, Santiago GMP, Pessoa ODL 2004. Chemical composition and larvicidal activity of the essential oils from Eupatorium betonicaeforme (D.C.) Baker (Asteraceae). J Agric Food Chem 52: 6708-6711.

Araújo ECC, Silveira ER, Lima MAS, Andrade-Neto M, Andrade IL, Santiago GMP, Mesquita ALM 2003. Insectidal activity and chemical composition of volatile oils from Hyptis martiusii. J Agric Food Chem 51: 3760-3762.

Chariandy CM, Seaforth CE, Phelps RH, Pollard GV, Kambay
BPS 1999. Screening of medicinal plants from Trinidad and Tobago for antimicrobial and insecticidal properties. J Ethnopharmacol 64: 265-270.

Cheng SS, Chang HT, Chang ST, Tsai KH, Chen WJ 2003. Bioactivity of selected plant essential oils against the yellow fever mosquito Aedes aegypti larvae. Bioresource Technol 89: 99-102.

Chun J, Goodman CL, Rice WC, Mcinthosi AH, Chippendale GM, Schubert KR 1994. Pentaclethra macroloba seed effect on larval growth cell viability and midgut enzyme activity of Helicoperva zea. J Econ Entomol 87: 1754-1760.

Ciccia G, Coussio J, Mongelli E 2000. Insecticidal activity against Aedes aegypti larvae of some medicinal South American plants. J Ethnopharmacol 72: 185-189.

Mohan S, Felds PG 2002. A simples technique to assess compounds that are reppelent or attractive to storedproducts insects. $J$ Stored Prod Res 38: 23-31.

Oliveira MF, Lemos TLG, Mattos MC, Segundo TA, Santiago GMP, Braz-Filho R 2002. New enamines derivatives of lapachol and biological activity. An Acad Bras Cienc 74: 311-221.

Pelah D, Abramovich Z, Wiesman MK 2002. The use of commercial saponin from Quillaga saponaria bark as a natural larvicidal agent against Aedes aegypti and Culex pipiens. J Ethnopharmacol 81: 407-409.

Santos RP, Viana FA, Lemos TLG, Silveira ER, Braz-Filho R, Pessoa, ODL 2003. Structure elucidation and total assignment of ${ }^{1} \mathrm{H}$ and ${ }^{13} \mathrm{C}$ nmr data for a new bisdemoside saponin from Cordia piauhiensis. Magn Reson Chem 41: 735-738.

Viana FA, Braz-Filho R, Pouliquen YBM, Andrade-Neto M, Santiago GMP, Rodrigues-Filho E 2004a. Triterpenoid saponins from stem bark of Pentaclethra macroloba. J Braz Chem Soc 15: 595-602.

Viana FA, Pouliquen YBM, Andrade-Neto M, Santiago GMP, Pessoa ODL, Rodrigues-Filho E, Braz-Filho $\mathrm{R}$ 2004b. Complete ${ }^{1} \mathrm{H}$ and ${ }^{13} \mathrm{C}$ assignments for two new monodesmoside saponins from Pentaclethra macroloba (Willd.) Kuntze. Magn Reson Chem 42: 695-699.

Yang YC, Lee SG, Lee HK, Kim MK, Lee SH, Lee HS 2002. A piperidine amide extracted from Piper longum L. fruit shows activity against Aedes aegypti mosquito larvae. J Agric Food Chem 50: 3765-3767. 